\title{
Offline Signature Verification Using Local Interest Points and Descriptors*
}

\author{
Javier Ruiz-del-Solar, Christ Devia, Patricio Loncomilla, and Felipe Concha \\ Department of Electrical Engineering, Universidad de Chile
}

\begin{abstract}
In this article, a new approach to offline signature verification, based on a general-purpose wide baseline matching methodology, is proposed. Instead of detecting and matching geometric, signature-dependent features, as it is usually done, in the proposed approach local interest points are detected in the signature images, then local descriptors are computed in the neighborhood of these points, and afterwards these descriptors are compared using local and global matching procedures. The final verification is carried out using a Bayes classifier. It is important to remark that the local interest points do not correspond to any signaturedependent fiducial point, but to local maxima in a scale-space representation of the signature images. The proposed system is validated using the GPDS signature database, where it achieves a FRR of $16.4 \%$ and a FAR of $14.2 \%$.
\end{abstract}

Keywords: Signature Verification, Matching techniques, SIFT descriptors.

\section{Introduction}

The use of signatures is one of the most employed mechanisms to verify the identity of individuals; the whole bank-check payment system is based on the use of signatures. In this context, computer-based signature verification is an active research area in biometry. Although signature-scan is a behavioral biometrics that depends on mood, fatigue, etc., several online and offline verification systems have been developed in the last years [10]-[18]. Online verification systems capture dynamic features such as time, pressure and speed during the writing process, while offline verification systems analyze static signature images, after the writing process. Offline signature verification has no dynamic information available, and therefore is a hardest process than online signature verification.

Most of the offline signature verification methods are based on the extraction of geometric, signature-dependent features from signature images, and their posterior classification. Examples of employed features are [13][18]: signature enveloped descriptors, interior stroke distribution descriptors, modified directional features, centroid features, tri-surface features, length features, six fold-surface features, best-fit features, etc. The classification step is normally implemented using Hidden Markov Models [11][13][15], neural networks [14][18], or statistical classifiers as SVM [12][13][15][17] or nearest-neighbor classifiers [13].

* This research was funded by Millennium Nucleus Center for Web Research, Grant P04-067-F, Chile. 
In this general context the main objective of this article is to propose a new approach to automatic signature verification that it is based on a general-purpose wide baseline matching methodology. Instead of detecting and matching standard geometric, signature-dependent features, in the proposed approach local interest points are detected in the signature images, then local descriptors are computed in the neighborhood of these points, and finally these descriptors are compared using local and global matching procedures. The final verification is carried out using a Bayes classifier. The local interest points do not correspond to any signature-dependent fiducial point, but to local maxima in a scale-space representation of the signature image (see examples in Figure 1).

Our main intention in proposing this new approach is to show an alternative procedure to tackle the signature verification process. We believe that this new approach can complement and enrich the standard procedures, and that it can be used in addition to them. In this sense, we are not proposing a methodology to replace the standard one, but a complementary solution.

This article is structured as follows. In section 2 the employed methodology for solving the general wide-baseline matching problem is described. In section 3 the adaptation of this methodology to signature verification is presented. In section 4 preliminary results of this new approach for signature verification are presented. Finally, in section 5 some conclusions and projections of this work are given.
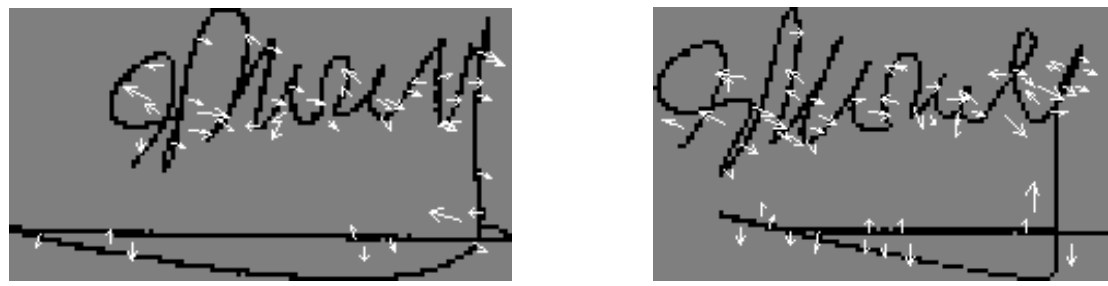

Fig. 1. Example of detected local interest points in a pair of test and template signature images. Interest points are displayed as arrows, whose origin, orientation and size corresponds to the position $(x, y)$, orientation $\theta$ and scale $s$ of the corresponding interest points.

\section{Wide Baseline Matching Using Local Interest Points}

Object recognition and wide baseline matching approaches based on local interest points have become increasingly popular and have experienced an impressive development in the last years [1][3][4][6][9]. Typically, local interest points are extracted independently from both a test and a reference image, then characterized by invariant descriptors, and finally the descriptors are matched until a given geometric transformation between the two images is obtained. Most employed local detectors are the Harris detector [2] and Lowe's sDoG+Hessian [3], being Lowe's detector multiscale and the Harris detector single scale. Best performing affine invariant detectors are the Harris-Affine and the Hessian-Affine [8], but they are too slow to be applied in general-purpose applications. The most popular and best performing invariant descriptor [7] is the SIFT (Scale Invariant Feature Transform) [3]. 
The local detectors and invariant descriptors to be used in a given application should be selected by taking into account their accuracy, robustness and processing speed. Lowe's system [3] using the SDoG+Hessian detector, SIFT descriptors and a probabilistic hypothesis rejection stage is a popular choice, given its recognition capabilities, and near real-time operation. However, Lowe's system main drawback is the large number of false positive detections. This is a serious problem when using it in real-world applications. One of the main weaknesses of Lowe's algorithm is the use of just a simple probabilistic hypothesis rejection stage, which cannot successful reduce the number of false positives. Loncomilla and Ruiz-del-Solar (L\&R) proposed a system that reduces largely the number of false positives by using several hypothesis rejection stages [4][5]. This includes a fast probabilistic hypothesis rejection stage, a linear correlation verification stage, a geometrical distortion verification stage, a pixel correlation verification stage, a transformation fusion procedure, and the use of the RANSAC algorithm and a semilocal constraints test. In [5] are compared the Lowe's and the L\&R systems using 100 pairs of real-world high-textured images (variations in position, view angle, image covering, partial occlusion, in-plane and out-of the-plane rotation). The results show that in this dataset the L\&R system reduces the false positive rate from $85.5 \%$ to $3.74 \%$, by increasing the detection rate by $5 \%$. For this reason we choose to use this system in this work.

The L\&R system considers four main stages: (i) Generation of local interest points, (ii) Computation of the SIFT descriptors, (iii) SIFT-matching using nearest descriptors, and (iv) Transformation Computation and Hypothesis Rejection Tests. The first three stages are the standard stages proposed by Lowe, while the fourth stage is employed for reducing the number of false matches, giving robustness to the whole system. This stage is implemented by the following procedure [4][5]:

1. Similarity transformations are determined using the Hough transform. After the Hough transform is computed, a set of bins, each one corresponding to a similarity transformation, is determined. Then:

a. Invalid bins (those that have less than 4 votes) are eliminated.

b. $Q$ is defined as the set of all valid candidate bins, the ones not eliminated in 1.a.

c. $R$ is defined as the set of all accepted bins. This set is initialized as a void set.

2. For each bin $B$ in $Q$ the following tests are applied (the procedure is optimized for obtaining high processing speed by applying less time consuming tests first):

a. If the bin $B$ has a direct neighbor in the Hough space with more votes, then delete bin $B$ from $Q$ and go to 2 .

b. Calculate $r_{R E F}$ and $r_{T E S T}$, which are the linear correlation coefficients of the interest points corresponding to the matches in $B$, that belong to the reference and test image, respectively. If the absolute value of any of these two coefficients is high, means that the corresponding points lie, or nearly lie, in a straight line, and that the affine transform to be obtained can be numerically unstable. If this condition is fulfilled delete bin $B$ from $Q$ and go to 2 .

c. Calculate the probability $P_{F A S T}$ associated to $B$. If $P_{F A S T}$ is lower than a threshold $P_{T H 1}$, delete bin $B$ from $Q$ and go to 2 . The main advantage of this probability test is that it can be computed before calculating the affine transformation, which speeds up the whole procedure. 
d.Calculate an initial affine transformation $T_{B}$ using the matches in $B$.

e. Compute the affine distortion degree of $T_{B}$ using a geometrical distortion verification test. A certain affine transformation should not deform very much an object when mapping it. Therefore, if $T_{B}$ has a strong affine distortion, delete bin $B$ from $Q$ and go to 2 .

f. Top down matching: Matches from all the bins in $Q$ that are compatible with the affine transformation $T_{B}$ are summarized and added to bin $B$. Duplication of matches inside $B$ is avoided.

g. Compute the Lowe's probability $P_{\text {LOWE }}$ of bin $B$. If $P_{L O W E}$ is lower than a threshold $P_{T H 2}$, delete bin $B$ from $Q$ and go to 2 .

h. To find a more precise transformation apply RANSAC inside bin $B$. In case that RANSAC success, a new transformation $T_{B}$ is calculated and $B$ is labeled as a RANSAC-approved bin.

i. Accept the candidates $B$ and $T_{B}$, what means delete $B$ from $Q$ and include it in $R$ (the $T_{B}$ transformation is accepted).

3. For all pairs $\left(B_{i}, B_{j}\right)$ in $R$, check it they may be fused into a new bin $B_{k}$. If the bins may be fused and one of them is RANSAC-approved, do not fuse them, and delete the other in order to preserve accuracy. If the two bins are RANSAC-approved, delete the least probable. Repeat this until all possible pairs (including the new created bins) have been checked.

4. For any bin $B$ in $R$, apply the semi-local constraints procedure to all matches in $B$. The matches from $B$ who are incompatible with the constraints are deleted. If some matches are deleted from $B, T B$ is recalculated.

5. For any bin $B$ in $R$, calculate the pixel correlation $r_{\text {pixel }}$ using $T B$. Pixel correlation is a measure of how similar are the image regions being mapped by $T B$. If $r_{\text {pixel }}$ is below a given threshold, delete $B$ from $R$.

6. Assign a priority to all bins (transformations) in R. The initial priority value of a given bin will correspond to its associated $P_{\text {LOWE }}$ probability value. In case that the bin is a RANSAC-approved one, the priority is increased in one. Thus, RANSACapproved bins have a larger priority than non RANSAC-approved ones.

\section{Proposed System for Signature Verification}

The proposed system for signature verification is based on the L\&R wide baseline matching system described in the former section. However, to apply this system in real-world, harsh conditions (state of the art signature testing protocols) we include a statistical classification post-processing module. Figure 2 shows a block diagram of the proposed signature verification system.

\subsection{Signature Verification Analysis}

In the first stage of our research work, we analyzed the suitability of the proposed approach. We carried several signature analysis experiments using different sets of parameters of the L\&R system and different signature public databases, and we verified that the $L \& R$ wide baseline system allows matching signature images. In Figure 3 we show exemplar experimental results. As it can be observed in these four examples, the wide baseline system achieves to match correctly the signature pairs. It 
can be verified that the local interest points do not correspond to standard geometric, signature-dependent features. As mentioned they correspond to local maxima in the position-scale multi-resolution representation of the signature images.

One of problems in signature verification is the nonlinear distortion in signature images, which disturbs the matching process. The here-proposed wide baseline matching approach is robust against these nonlinear distortions. It can be proved that any differentiable non-linear transformation that is locally near-orthogonal can be approximated by a bundle of local similarity approximations using the Hough transform methodology, if the density of matches between interest points is enough high (see proof in [19]).

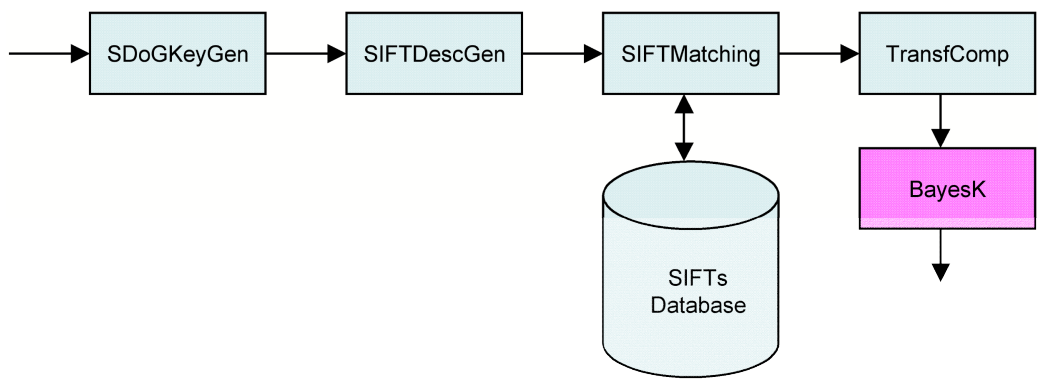

Fig. 2. Block diagram of the proposed signature verification system. SDoGKeyGen: SDoG Keypoints Generation; SIFTDescGen: SIFT Descriptors Generation; TrasfComp: Transformation Computation and hypothesis rejection tests; BayesK: Bayes Classifier.

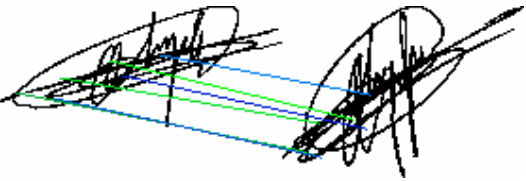

(a)

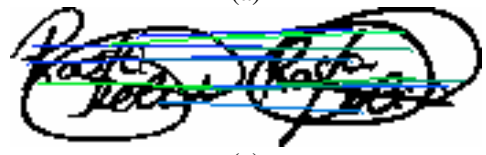

(c)

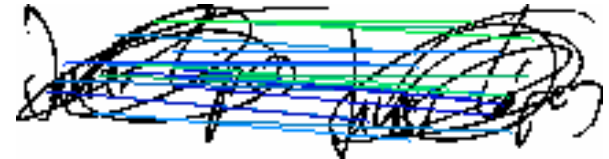

(b)

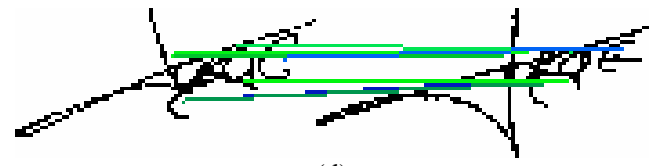

(d)

Fig. 3. (a)-(d) Signature reference-test image pairs with matches

\subsection{Bayes Classification}

When applying the L\&R methodology in signature verification cases when skilled forgeries are analyzed or when the signature's quality changes largely (changes in signature position, orientation, etc.), we noted that the number of false positives was large. We solve this problem by applying a statistical classifier (Naïve Bayes) after the wide baseline matching original system. As in [18], we train a statistical classifier of each individual. We define the following 12 features for the classifier, whose relevance is afterwards analyzed: 
- TNMatches: Total number of SIFT matches between the reference and test images.

- PTime: Processing time as a measure of the complexity of the matching process.

- NAffinT: Number of detected affine transformations between the reference and test images.

- NMatches: Number of associated SIFT matches included in the best transformation.

- PBT: Probability of the best transformation

- LCorr: Linear correlation parameter of the best transformation

- PCorr: Pixel correlation parameter of the best transformation

- MNDesc: Maximum number of test image descriptors who are matched to the same reference image descriptor, considering the best transformation.

- ScaleAffinMax: Absolute value of the largest eigenvalue of the affine transformation matrix of the best transformation (i.e. largest scale of the best affine transformation).

- ScaleAffinMin: Absolute value of the lowest eigenvalue of the affine transformation matrix of the best transformation (i.e., lowest scale of the best affine transformation).

- NIncMatches: Number of matches of the best transformation who are incompatible with the semi-local constraints.

- RansacPar: RANSAC-compatibility with precise affine transform. A small subset of 3 matches from B is selected to construct a transformation TB who is tested against all the resting matches in B. The matches from B who are correctly mapped using TB with a very little error are called compatible matches. If more than $2 / 3$ of the matches from B are compatible, the incompatible matches are deleted and a final fine transformation is calculated using the compatible matches. This procedure is tried 10 times using different subsets with 3 matches from $\mathrm{B}$. If in none of the 10 iterations a fine transformation is obtained, RANSAC fails and the initial transformation is preserved.

We analyzed the relevance of these features using the Weka package [20]. With the Weka's BestFirst attributes selection method, which searches the space of attribute subsets by greedy hill-climbing, we selected the final attributes that we use in each of the classifiers.

\section{Preliminary Results}

We present some preliminary results of the operation of the proposed system for signature verification. First, the system is tuned using signature images obtained in our laboratory. Then, the system is validated using the GPDS signature database [21], one of the very few available for public use. This database "contains data from 160 individuals: 24 genuine signatures for each individual, plus 30 forgeries of his/her signature. The 24 genuine specimens of each signer were collected in a single day writing sessions. The forgeries were produced from the static image of the genuine signature. Each forger was allowed to practice the signature for as long as s/he wishes. Each forger imitated 3 signatures of 5 signers in a single day writing session. The genuine signature shown to each forger is chosen randomly from the 24 genuine ones. Therefore for each genuine signature there are 30 skilled forgeries made by 10 forgers from 10 different genuine specimens" [21]. 
As in [18], we tested our verification system using randomly selected signatures from 44 individuals. Our experiments considered genuine-genuine matches, genuine random forgery matches (a genuine signature is compared against a signature from other randomly taken user), and genuine - skilled forgery matches (a genuine signature is compared against an own forgery). For each user, a Naïve Bayes classifier of the proposed signature verification system was trained using the Weka package [20] and 10-fold cross-validation. Previously for each classification the relevant features were automatically selected using Weka. Table 1 shows the training and test sets employed for our experiments.

The Bayesian classifier helps to discard a great amount of false detections, which are produced by the signature local symmetry. The results of the final verification system are FRR of $16.4 \%$ and a FAR of $14.2 \%$. It is important to mention that previous works that have used the GPDS signature database have simplified the problem either by non-using skilled forgeries [13], or by using a very reduced amount of skilled forgeries in the experiments; 25 from 889 (2.8\%) comparisons/ experiments in [18], compared to 500 from $1440(34.7 \%)$ in our case. If this fact is taken into account, it can be seen that our results are comparable to the ones reported in [13] and [18]. However, it should be stressed that our results are obtained without using any information about the nature of images' content (signature information).

Table 1. Training and test sets for the experiments

\begin{tabular}{|l|l|l|}
\hline Matching Experiments & Training & Test \\
\hline Genuine-Genuine & 190 & 80 \\
\hline Genuine - Random Forgery & 172 & 860 \\
\hline Genuine - Skilled Forgery & 100 & 500 \\
\hline
\end{tabular}

\section{Conclusions}

A new approach to automatic signature verification based in a general-purpose wide baseline matching methodology was proposed. Instead of detecting and matching the standard structural features, in the proposed approach local interest points are detected in the signature images, then local descriptors are computed in the neighborhood of these points, and finally these descriptors are matched. Several verification stages and a simple statistical classifier are employed for reducing the number of false positives. The proposed signature verification system was validated using the GPDS signature database. Without using any a priori knowledge information about the nature of images' content (signature information), the system achieves a FRR of $16.4 \%$ and a FAR of $14.2 \%$. We believe that the obtained results are encouraging, and that the here-proposed procedure for solving the signature verification process can complement and enrich the standard procedures, and it can be used in addition to them.

\section{References}

1. Ferrari, V., Tuytelaars, T., Van Gool, L.: Simultaneous Object Recognition and Segmentation by Image Exploration. In: Pajdla, T., Matas, J. (eds.) ECCV 2004. LNCS, vol. 3021, pp. 40-54. Springer, Heidelberg (2004) 
2. Harris, C., Stephens, M.: A combined corner and edge detector. In: Proc. 4th Alvey Vision Conf., pp. 147-151. Manchester, UK (1998)

3. Lowe, D.: Distinctive Image Features from Scale-Invariant Keypoints. Int. Journal of Computer Vision 60(2), 91-110 (2004)

4. Loncomilla, P., Ruiz-del-Solar, J.: Gaze Direction Determination of Opponents and Teammates in Robot Soccer. In: Bredenfeld, A., Jacoff, A., Noda, I., Takahashi, Y. (eds.) RoboCup 2005. LNCS (LNAI), vol. 4020, pp. 230-242. Springer, Heidelberg (2006)

5. Loncomilla, P., Ruiz-del-Solar, J.: A Fast Probabilistic Model for Hypothesis Rejection in SIFT-Based Object Recognition. In: Martínez-Trinidad, J.F., Carrasco Ochoa, J.A., Kittler, J. (eds.) CIARP 2006. LNCS, vol. 4225, pp. 696-705. Springer, Heidelberg (2006)

6. Mikolajczyk, K., Schmid, C.: Scale \& Affine Invariant Interest Point Detectors. Int. Journal of Computer Vision 60(1), 63-96 (2004)

7. Mikolajczyk, K., Schmid, C.: A performance evaluation of local descriptors. IEEE Trans. Pattern Anal. Machine Intell. 27(10), 1615-1630 (2005)

8. Mikolajczyk, K., Tuytelaars, T., Schmid, C., Zisserman, A., Matas, J., Schaffalitzky, F., Kadir, T., Van Gool, L.: A Comparison of Affine Region Detectors. Int. Journal of Computer Vision 65(1-2), 43-72 (2005)

9. Schaffalitzky, F., Zisserman, A.: Automated location matching in movies. Computer Vision and Image Understanding 92(2-3), 236-264 (2003)

10. Leclerc, F., Plamondon, R.: Automatic Signature Verification: The State of the Art 19891993. Int. J. Pattern Recognition and Artificial Intelligence, Special Issue on Signature Verification 8(3), 643-660 (1984)

11. Edson, J., Justino, R., Bortolozzi, F., Sabourin, R.: An Off-Line Signature Verification Using HMM for Random, Simple and Skilled Forgeries. In: Proc. Sixth Intl. Conf. Document Analysis and Recognition, pp. 1031-1034. IEEE Press, Washington (2001)

12. Martínez, L.E., Travieso, C.M., Alonso, J.B., Ferrer, M.A.: Parameterization Method of a Forgery Handwritten Signature Verification System Using SVM. In: Proc. 38th IEEE Int'1 Carnahan Conf. Security Technology, pp. 193-196 (2004)

13. Ferrer, M.A., Alonso, J.B., Travieso, C.M.: Offline geometric parameters for automatic Signature Verification using fixed-point arithmetic. IEEE Trans. on Pattern Anal. Machine Intell. 27(6), 993-997 (2005)

14. Huang, K., Yan, H.: Off-Line Signature Verification Based on Geometric Feature Extraction and Neural Network Classification. Pattern Recognition 30(1), 9-17 (1997)

15. Justino, E.J.R., Bortolozzi, F., Sabourin, R.: A comparison of SVM and HMM classifiers in the off-line signature verification. Pattern Recognition Letters 26, 1377-1385 (2005)

16. Kalera, M.K., Srihari, S., Xu, A.: Off-line signature verification and identification using distance statistics. Int. J. of Patt. Recog. and Artificial Intelligence 18(7), 1339-1360 (2004)

17. Lv, H., Wang, W., Wang, C., Zhuo, Q.: Off-line Chinese Signature Verification based on Support Vector Machines. Pattern Recognition Letters 26, 2390-2399 (2005)

18. Armand, S., Blumenstein, M., Muthukkumarasamy, V.: Off-line Signature Verification Using an Enhanced Modified Direction Feature with Single and Multi-classifier Approaches. IEEE Computational Intelligence Magazine, 18-25 (2007)

19. Ruiz-del-Solar, J., Loncomilla, P., Devia, C.: A New Approach for Fingerprint Verification based on Wide Baseline Matching using Local Interest Points and Descriptors. In: Mery, D., Rueda, L. (eds.) PSIVT 2007. LNCS, vol. 4872, pp. 586-599. Springer, Heidelberg (2007)

20. Weka website, http://www.cs.waikato.ac.nz/ml/weka/

21. GPDS Signature Verification database, http: / / www. gpds.ulpgc.es / download/index.htm 